machines arrange vaccination and immunization programmes of some public health departments, and their vast possibilities in the field of biochemical laboratory measurements are becoming apparent. Autoanalytical methods are well developed for sampling blood plasma for electrolytes and are in use in most hospital laboratories. The results from these instruments are produced on automatic pen-recorded charts. Data from the charts can be inserted directly into the computer by a device that measures the height of the curves at intervals and converts each height into a coded row of holes on continuous paper tape. If a suitable programme is prepared the computer will then analyse and record the results. If previous biochemical records of the patient have been stored in the computer they can then be sought and retrieved from the store. With the number of hospital biochemical investigations doubling every five years such automation must obviously be exploited to the full.

Computers are playing a part in planning radiation treatment. The delivery of the optimum highest dosage to tumours while subjecting normal tissues to the minimum dose requires much work in the planning of individual treatments. An accurate assessment of differing absorption in fat, air, and bone demands much tedious and time-consuming work. By developing a computer programme using the knowledge of the most experienced physicist it is possible for less-informed iunior staff to produce a plan of treatment.

The assistance of computers has, however, never been more essential in medical practice than in the investigation and diagnosis of cardiovascular disease. The assessment of its role in cardiovascular investigation by S. H. Taylor and his colleagues in a recent issue of the British Heart fournal is therefore timely. ${ }^{1}$ The applications of computers include epidemiological studies, E.C.G. diagnosis, cardiovascular diagnostic investigations, and the continuous monitoring of patients with severe myocardial infarction. Indicator-dilution techniques for measuring cardiac outputs have been so automated that dilution curves can be produced by automatic injection and the sampling of arterial blood at any desired frequency and for any length of time. The information produced by these methods is overwhelming, for each hour spent in investigating provides more than 80 hours of work by skilled staff in assessing the results. This is not only timeconsuming but intellectual imprisonment. Computer-assisted analysis offers an obvious solution. The curve from the automatic pen-recorded charts of cardiac output can be prepared for insertion into the computer in a similar way to biochemical autoanalytical data.

Taylor and his colleagues have been in the forefront of the application of computer programmes to cardiovascular problems. They have prepared computer programmes for cardiovascular investigations and have surmounted many of the problems presented by the computer's ineptitude at pattern recognition. They have assessed the precision with which three computers measure the cardiac output in man and have determined their usefulness in cases of abnormalities of cardiac rhythm and valvular disease. As well as showing how cardiac investigations may be advanced they have given an important lead in surmounting the many difficulties caused by artifacts in the tracings. Their work is a reminder that the potential of computers in the medical field is still largely untapped and it should stimulate the application of them to other branches of medicine.

\section{Testing Drugs for Rheumatoid Arthritis}

The medical treatment of rheumatoid arthritis is based on measures to reduce the pain, heat, redness, swelling, and inflammation. The pharmaceutical industry devotes vast resources to discovering new drugs for this purpose. Promising compounds are screened by animal tests. Those few which pass have still to be tested in man, since there is no guarantee that ability to check experimental inflammation in the rat implies ability to check rheumatoid inflammation in the human joint. But more effective anti-inflammatory drugs are needed, for the preparations at present available have many deficiencies, and there are always patients who fail to respond to one drug and yet respond to another. Any effective new preparation, even if not specially remarkable in itself, increases the pool of reserve drugs which can be brought into play.

Facilities for testing anti-inflammatory drugs in human disease are not easily found, nor are the doctors with both the will and the skill to do the task. Thus clinical testing is, to borrow the pharmacologists' jargon, the "rate-limiting factor" in the availability of new anti-inflammatory drugs. For this reason the paper by Drs. P. L. Boardman and F. Dudley Hart at page 264 of the B.M.7. this week is of practical importance in that it describes methods which are simple, reliable, and reproducible for assessing antiinflammatory drugs in rheumatoid arthritis. These methods can be used in outpatient departments in any hospital or rheumatism clinic. They consist in a short-term, controlled trial, with alternate or random allocation of patients to an initial treatment group and then crossing-over to the other group. Measurements of heat and redness in rheumatoid arthritis being unreliable, Boardman and Hart concentrated their attention on measurements of swelling and function. They first used jewellers' rings to measure swelling in the finger 16 years ago. ${ }^{1}$ But they have been able to show that a small instrument which measures the circumference of the joint directly is equally reliable and much more convenient for routine use. Strength of grip is another method long used for measuring hand function. A modified sphygmomanometer cuff of prescribed dimensions is inflated and connected to a mercury manometer. In practice strength of grip reflects both pain and structural dysfunction, but chiefly pain. Provided these methods are used at precisely the same time of day and the same time after giving the drug, they are now so reliable and reproducible that they deserve internationally agreed standardization.

Boardman and Hart found-as others have done-that placebos may apparently be followed both by " toxic" reactions and by apparent therapeutic benefit. Doctors untrained in mathematics used to boggle at the statistics involved in trials. Nowadays the path is eased by the technique of sequential analysis ${ }^{2}$ and the now familiar "pair of trousers" charts, ${ }^{3}$ by which preferences between paired observations can be entered on the chart and the trial stopped as soon as a result unlikely to have happened by chance has been obtained. Using such methods, Boardman and Hart show that prednisolone has an undoubted anti-inflammatory action, that paracetamol has little, if any, anti-inflammatory action, and that salicylate in low dosage can relieve the pain of inflammation

1 Hart, F. D., and Clark, C. J. M., Lancet, 1951, 1, 775.

Bross, I., Biometrics, 1952, 8, 188. Taylor, S. H., Macdonald, H. R., Robinson, M. D., and Sapru, R. P.,
Brit. Heart f., 1967, 29, 352 . 
but that high dosage is needed to affect the swelling. Their paper exemplifies methods which clinicians could use more widely for the clinical testing of potentially useful new antiinflammatory drugs.

\section{No Need for Change?}

The Minister of Health in H.M. (67) 58 has told members of hospital boards and committees that he does not accept the need for fundamental changes in methods of training nurses proposed by the Royal College of Nursing in its publication $A$ Reform of Nursing Education. ${ }^{12}$ The Minister believes that improvements can be produced by the provision of more tutors or better use of those available, by better selection of students and possibly the streaming of entrants, and by careful attention to the principles of student training.

Nurse administrators will read the form of self-examination suggested for their use somewhat glumly. Nothing is suggested that they have not often thought about before, but perhaps committees will listen with more attention to the Minister than they have to nurse advisers. In addition, hospitals will now be required to give returns of their student wastage, and, while this may be thought to amount to examining the stable door after the horse has bolted, the information ought to be available from all training schools.

The last section of the memorandum ends with suggestions for experimental schemes of nurse training. Hitherto, training schools have submitted such schemes to the General Nursing Council, which has sent those approved to the Ministry for ratification, and the Council has had many such schemes to consider. The Minister's list contains one new idea-that students might attend colleges of further education, presumably on a day-release basis, for " liberal studies."

At the same time as H.M. (67) 58 was published the General Nursing Council received from the students of a nurse-training school a letter which throws doubts on the validity of the Minister's belief that all is fundamentally well in nursing schools. Here is an extract:

\footnotetext{
"We wonder whether any of you know under what sort of conditions we are working here due to chronic shortage of staff. . . . This hospital seems to be staffed only by student nurses and a few sisters. The student nurses do not get any teaching experience on the ward due to the shortage of time and trained staff. One leaves a ward after three months' drudgery without ever being taught anything whatsoever. Does one attempt to do a procedure properly, we are very often told there is not time to do a sterile procedure. . . . Still exhausted from the previous day, we often find ourselves alone on the ward, dishing out breakfast for 28 patients, feeding many of them as well. . . Night duty follows a very similar pattern. One is put alone in charge of a 28-patient ward without previous experience of night duty, giving out drugs, or general supervision of patients."
}

The full text was published in the Nursing Times ${ }^{3}$ recently and a copy was sent to the Minister. It reminds us that nobody and no organization concerned with nurse training can be satisfied with the present position.

\footnotetext{
A Reform of Nursing Education. Royal College of Nursing, 1964. London.

Brit. med. 7., 1964, 1, 1585

3 Nursing Times, 22 Septembe: 1967
}

\section{Modernizing Hospital Medicine}

One of the more depressing features of British medicine for those who believe in the N.H.S. is the antagonism between the profession and the Health Ministers. It is, therefore, all the more encouraging this week to find representatives of the two sides agreeing on the major faults in the organization of medical work in the hospital service. Just as welcome are their suggestions for reform, for these are both radical and realistic.

A joint working party of doctors and hospital administrators was set up by the Secretary of State for Scotland in 1965 with instructions to look for ways of improving efficiency in the hospital service. A little later a similar body was set up by the Ministry of Health and the Joint Consultants' Committee. Their first reports ${ }^{12}$ are summarized at page 291 of this week's B.M.F.

Fundamental to future planning for the hospital service is the knowledge that there are too few doctors and that there is no prospect of enough for at least ten years. So doctors must be used more efficiently, and their morale must be improved if they are not to emigrate. The Scottish report accepts that the discrepancy between the number of registrars and the number of potential consultant vacancies is responsible for much anxiety among junior staff, and recommends that numbers in the registrar grade should be reduced to the minimum which would allow for natural wastage and competition. The long-term solution to much of the discontent lies at this intermediate level of hospital work, where there is need for greater integration with general practice. The Scottish Working Party believes that much of the work could be done by family doctors if they could be brought into the hospitals as, say, part-time medical assistants. The report recognizes that expansion of the medical assistant grade has dangers, particularly the risk of consultant responsibility being carried by assistants-and it accepts that the present sessional rates of pay are not good enough to attract general practitioners. Such an appreciation of realities is welcome, as is the Working Party's conclusion that "these difficulties must be resolved however intractable they may seem."

Both reports agree that the tradition in hospitals of consultants having a fixed allocation of beds is inefficient and out of date. Both propose that hospitals should change over to a divisional system of staffing, similar to that widely used in North America. A division would consist of all the doctors working in one specialty-or in several specialties if they are linked in their functions. Chairmen of divisions would be appointed, and a committee formed of these chairmen or their representatives. Outside Scotland the chairman of this committee would hold a post roughly equivalent to the American chief of staff; in Scotland the position of medical superintendent would be retained.

These changes should lead to a greater exchange of ideas between medical staff, as well as promoting the more efficient use of beds, staff, and equipment. The danger is that a doctor's freedom to treat his patients as he thinks best may be eroded by pressures for the adoption of uniform regimens of management. Neither report even hints at the introduction of sanctions that could be used to force the nonconformist into obedience, though these do exist in certain American hospitals. The Scottish report states, "While we do not wish to depart from the principle that each

\footnotetext{
Organization of Medical Work in the Hospital Service in Scotland 1967. H.M.S.O. Price 6s. 3d.

First Report of the foint Working Party on the Organization of Medical Work in Hospitals, 1967. H.M.S.O. Price 2s. 9d.
} 